\title{
A Flare of Lupus Nephritis in an 11-Year-Old SLE Patient with a Mild COVID-19-Associated Pneumonia
}

\author{
Morteza Mousavi-Hasanzadeh ${ }^{1}$, Seyed Amir Sanatkar ${ }^{2}$, Manijeh Kahbazi ${ }^{3}$, Parsa Yousefichaijan ${ }^{4}$, \\ Amirreza Najmi ${ }^{5}$ and Yazdan Ghandi ${ }^{4, *}$ \\ ${ }^{1}$ Faculty of Medicine, Arak University of Medical Sciences, Arak, Iran \\ ${ }^{2}$ Department of Surgery, Arak University of Medical Sciences, Arak, Iran \\ ${ }^{3}$ Pediatric Infectious Disease, Amir Kabir Hospital, Arak University of Medical Sciences, Arak, Iran \\ ${ }^{4}$ Associated Professor, Department of Cardiology, Amir Kabir Hospital, Arak University of Medical Sciences, Arak, Iran \\ ${ }^{5}$ Infectious Disease Research Center, Arak University of Medical Sciences, Arak, Iran \\ "Corresponding author: Amir Kabir Hospital, Arak University of Medical Sciences, Arak, Iran. Email: drghandi1351@gmail.com
}

Received 2020 August 10; Accepted 2020 August 14.

\begin{abstract}
Children with immunological diseases like SLE are vulnerable to any type of infection, and their management is considered an important issue in the COVID-19 pandemic. Here, we describe an 11-year-old female, previously known to have lupus nephritis, complaining of tachypnea, persistent fever, low oxygen saturation $\left(\mathrm{SaO}_{2}\right)$, and generalized edema with confirmed COVID-19 using sputum PCR testing on the first day of admission.
\end{abstract}

Keywords: Children, COVID-19, SLE, Nephritic Syndrome

\section{Introduction}

Since December 2019, a novel coronavirus (COVID-19) emerged in Wuhan, China. It has involved almost all countries and emerged a new global pandemic (1). The rate of infection in children is reported to be low and milder in course, but children of any age are vulnerable to this virus, and they are known to be mostly a carrier (2). The management of patients with rheumatological disorders, such as systemic lupus erythematosus (SLE), who are prone to severe infections (3) is one of the new challenges in this pandemic for healthcare providers. Here, we describe a COVID19-infected child who already suffered from lupus nephritis.

\section{Case Presentation}

An 11-year-old female (weighing $43 \mathrm{~kg}$ ) previously diagnosed with lupus nephritis presented with five days of tachypnea, bilateral flank pain, persistent fever, low oxygen saturation $\left(\mathrm{SaO}_{2}\right)$ generalized edema. She was then admitted to a Pediatric Intensive Care Unit (PICU). Two years before this episode, she had been diagnosed with
SLE (lupus nephritis); since then, she has been taking hydroxychloroquine (HQC $200 \mathrm{mg} /$ day) plus prednisolone (5 $\mathrm{mg}$ /day) as standard treatment.

Her vital signs and laboratory tests on the day of admission are shown in Table 1 . In an examination, we only detected generalized edema. Because of persistent fever, tachypnea, and low $\mathrm{SaO}_{2}$, and considering the new pandemic of COVID-19 and immune suppression state of the patient, COVID-19 was a suspected diagnosis. Thus, a real-time reverse transcription-polymerase chain reaction (rRT-PCR) test was performed using nasal/pharyngeal swab samples, which showed positive. Baseline CXR (Figure 1A) was normal, while the results of the CT scan showed bilateral ground-glass opacities with some consolidation (Figure 1B). Because of the flank pain, abdominal ultrasonography was requested, which showed normal. To rule out cardiac involvement due to the prolonged HQC use, the patient underwent bedside echocardiography (Echo) and 12lead electrocardiogram (ECG), which showed normal.

The medical management of the patient included HQC (75 mg/TDS) and prednisolone (10 mg/TDS) plus antibiotics meropenem (80 mg/TDS), clindamycin (45 mg/TDS), and azithromycin (250 mg daily). The patient was hospitalized 

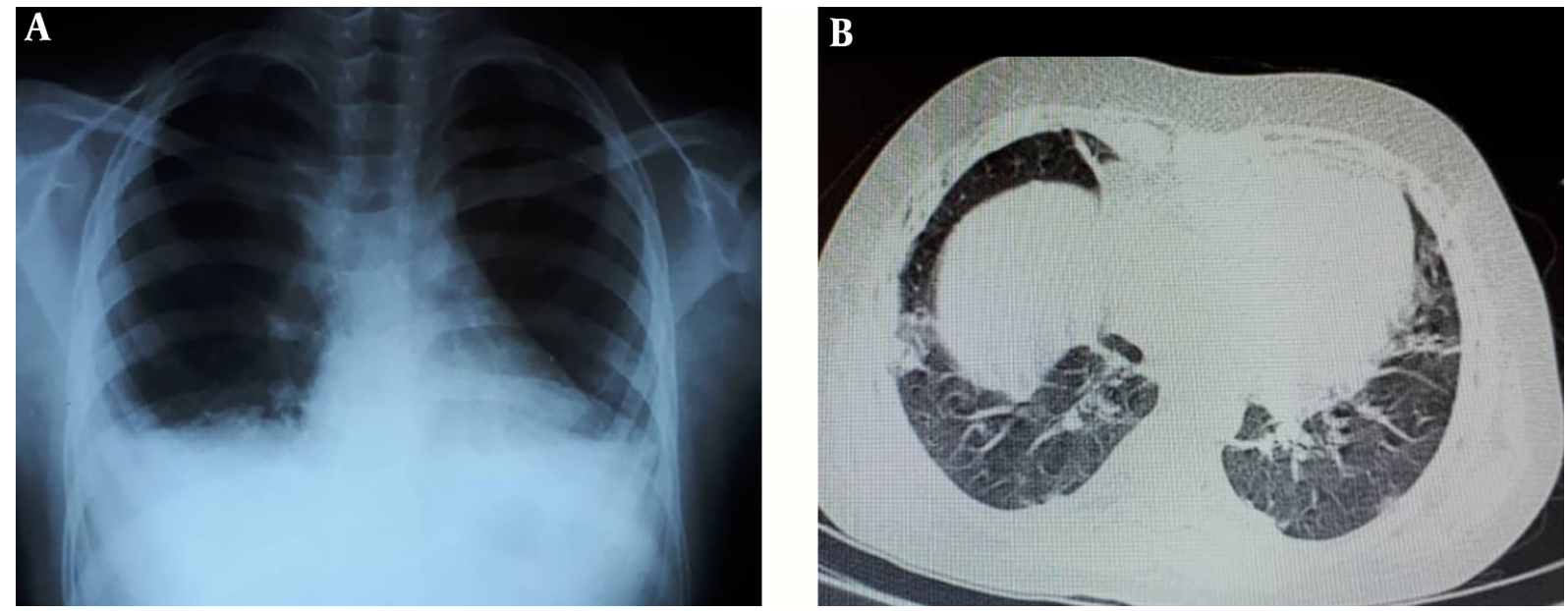

Figure 1. Radiological findings: A, CXR does not show any COVID-19 patterns; B, the chest CT scan reveals bilateral ground-glass opacities with consolidation

in the PICU for a day. Then, she was transferred to an isolated ward.

\section{Discussion}

Here, we presented an 11-year-old female with two years of lupus nephritis who developed COVID-19 pneumonia during treatment. The girl received oral HQC $200 \mathrm{mg} /$ day and prednisone $5 \mathrm{mg}$ /day since the diagnosis.

Viral infections are associated with an acceleration of rheumatological disorders (4); that is why we expect that initial symptoms of COVID-19 in SLE individuals might be very severe. This patient presented with persistent fever and dyspnea that were similar to the presentations of COVID-19 among previously healthy individuals (5). Thus, it seems that the presentations of COVID-19 might be the same in SLE patients on the immunosuppressive regimen and COVID-19 patients. It was confirmed in the study of adult SLE and COVID-19 patients by Mathian et al. (3). After a day of admission in the PICU, the patient declared better feelings and was transferred to an isolated ward. It was expected that COVID-19, in this case, would present with a severe course, as SLE patients are usually on immunosuppressive treatment (4). However, as data regarding the pattern of COVID-19 in SLE patients is not well known, the outcome in the management of such patients seems unpredictable.

This patient was on treatment with HQC and prednisone for approximately two years before the COVID-19 pandemic. A recent clinical trial by Gautret et al. (6) reported that HQC significantly reduced the viral load in
COVID-19. Also, HQC might have some therapeutic (7) and prophylactic (8) effects on COVID-19. According to the findings by Mathian et al. (3) showing that only two SLE cases died of COVID-19 pneumonia and considering that the fever and dyspnea were completely resolved after just one day, it seems that even though the use of HQC cannot prevent COVID-19, it is likely to reduce the severity of pneumonia induced by COVID-19. Accomplishing a definite finding in such a hypothesis needs future studies to be clearly understood.

On the other hand, the patient developed an episode of Acute Kidney Injury (AKI) and severe proteinuria, which had been controlled before. Recently, Larsen et al. (9) and Peleg et al. (10) reported cases of AKI in COVID-19. Besides, the Mathian et al. (3) study reported that $18 \%$ of SLE individuals who tested positive for COVID-19 developed AKI. It seems that COVID-19 in SLE patients who had been diagnosed as lupus nephritis may induce a flare of nephritis. These results may indicate that in addition to respiratory failure, acute renal failure should be considered in such groups.

In conclusion, the initial presentation of COVID-19 might be the same among SLE patients and other patients, and the HQC use in such individuals may decrease the severity of COVID-19-associated pneumonia. Also, COVID-19 may induce a flare of lupus nephritis among SLE patients; that is why the possibility of acute renal failure development should be considered in these individuals. 


\begin{tabular}{|c|c|}
\hline & Findings \\
\hline \multicolumn{2}{|l|}{ Vital sign } \\
\hline $\mathrm{BP}$ & $110 / 65$ \\
\hline PR & $110-120$ \\
\hline $\mathrm{RR}$ & $21-28$ \\
\hline Body temperature & 38.8 \\
\hline $\mathrm{O}_{2}$ sat, $\%$ & $89-90$ \\
\hline \multicolumn{2}{|l|}{ Blood test } \\
\hline \multicolumn{2}{|l|}{$\mathrm{CBC}$} \\
\hline $\mathrm{WBC}, \times 10^{9}$ cells $/ \mathrm{L}$ & 4.7 \\
\hline PMN, \% & 71 \\
\hline Lymp, \% & 24 \\
\hline $\mathrm{Hb}, \mathrm{g} / \mathrm{L}$ & 10.7 \\
\hline Plt, $\times 10^{9} / \mathrm{L}$ & 90 \\
\hline \multicolumn{2}{|l|}{ ABG } \\
\hline $\mathrm{PH}$ & 7.51 \\
\hline $\mathrm{HcO}_{3}, \mathrm{mmol} / \mathrm{L}$ & 19.3 \\
\hline $\mathrm{PcO}_{2}, \mathrm{mmHg}$ & 25 \\
\hline $\mathrm{BE}, \mathrm{mmol} / \mathrm{L}$ & -2.3 \\
\hline $\mathrm{CPK}, \mathrm{U} / \mathrm{L}$ & 25 \\
\hline $\mathrm{LDH}, \mathrm{U} / \mathrm{L}$ & 630 \\
\hline ALB, g/L & 3 \\
\hline Urea & 35 \\
\hline $\mathrm{Cr}$ & 1.33 \\
\hline ESR & 50 \\
\hline CRP & $1+$ \\
\hline \multicolumn{2}{|l|}{ Urine test } \\
\hline WBC & $3-5$ \\
\hline RBC & $20-25$ \\
\hline Blood & 1 plus \\
\hline Protein & 4 plus \\
\hline Urine culture & Negative \\
\hline
\end{tabular}

Abbreviations: ABG, blood gas analysis; BE, base excess; $\mathrm{Cr}$, creatinine; CRP, Creactive protein; ESR, erythrocyte sedimentation rate; $\mathrm{Hb}$; hemoglobin, $\mathrm{LDH}$ lactate dehydrogenase; Lymph, lymphocyte; $\mathrm{O}_{2}$ sat, oxygen saturation; PMN neutrophil count; WBC; white blood cell count. the report.

Conflict of Interests: The authors declare that they have no competing interests concerning the authorship and/or publication of this article.

Funding/Support: The Vice-Chancellor of Research and Technology of Arak University of Medical Sciences funded the study. The funder had no role in data collection, analysis, interpretation, and manuscript drafting.

Informed Consent: Informed consent was obtained from the parents of the patient.

\section{References}

1. Wu Z, McGoogan JM. Characteristics of and important lessons from the coronavirus disease 2019 (COVID-19) outbreak in China: summary of a report of 72314 cases from the Chinese Center for Disease Control and Prevention. Jama. 2020;323(13):1239-42.

2. Dong Y, Mo X, Hu Y, Qi X, Jiang F, Jiang Z, et al. Epidemiology of COVID19 among children in China. Pediatrics. 2020;145(6).

3. Mathian A, Mahevas M, Rohmer J, Roumier M, Cohen-Aubart F, Amador-Borrero B, et al. Clinical course of coronavirus disease 2019 (COVID-19) in a series of 17 patients with systemic lupus erythematosus under long-term treatment with hydroxychloroquine. Annals of the Rheumatic Diseases. 2020;79(6):837-9.

4. Chattopadhyay A, Mishra D, Sharma V, Naidu GSK, Sharma A. Coronavirus disease-19 and rheumatological disorders: A narrative review. Indian J Rheumatol. 2020;15(2):122-9.

5. Huang C, Wang Y, Li X, Ren L, Zhao J, Hu Y, et al. Clinical features of patients infected with 2019 novel coronavirus in Wuhan, China. The lancet. 2020;395(10223):497-506.

6. Gautret P, Lagier J, Parola P, Meddeb L, Mailhe M, Doudier B, et al. Hydroxychloroquine and azithromycin as a treatment of COVID-19: results of an open-label non-randomized clinical trial. International journal of antimicrobial agents. 2020:105949.

7. Yao X, Ye F, Zhang M, Cui C, Huang B, Niu P, et al. In vitro antiviral activity and projection of optimized dosing design of hydroxychloroquine for the treatment of severe acute respiratory syndrome coronavirus 2 (SARS-CoV-2). Clinical Infectious Diseases. 2020.

8. Pagliano P, Piazza O, De Caro F, Ascione T, Filippelli A. Is Hydroxychloroquine a possible post-exposure prophylaxis drug to limit the transmission to health care workers exposed to COVID19. Clin Infect Dis. 2020;24.

9. Larsen CP, Bourne TD, Wilson JD, Saqqa O, Moh'd A S. Collapsing glomerulopathy in a patient with coronavirus disease 2019 (COVID19). Kidney international reports. 2020.

10. Peleg Y, Kudose S, D’Agati V, Siddall E, Ahmad S, Kisselev S, et al. Acute kidney injury due to collapsing glomerulopathy following COVID-19 infection. Kidney International Reports. 2020.

\section{Footnotes}

Authors' Contribution: PY, YGH, and MMH designed the study and did the literature search. MK and YGH were responsible for disease diagnosis and treatment. MK, MMH, AN, and PY were responsible for data collection. PY, MMH, and YGH analyzed the data. All authors drafted and revised 